\title{
Inadequacy of zero-width approximation for a light Higgs boson signal
}

\author{
Nikolas Kauer \\ Department of Physics, Royal Holloway, University of London, Egham Hill, Egham \\ TW20 0EX, United Kingdom
}

\begin{abstract}
The zero-width approximation (ZWA) restricts the intermediate unstable particle state to the mass shell and, when combined with the decorrelation approximation, fully factorizes the production and decay of unstable particles. The ZWA uncertainty is expected to be of $\mathcal{O}(\Gamma / M)$, where $M$ and $\Gamma$ are the mass and width of the unstable particle. We review the ZWA and demonstrate that errors can be much larger than expected if a significant modification of the Breit-Wigner lineshape occurs. A thorough examination of the recently discovered candidate Standard Model Higgs boson is in progress. For $M_{H} \approx 125 \mathrm{GeV}$, one has $\Gamma_{H} / M_{H}<10^{-4}$, which suggests an excellent accuracy of the ZWA. We show that this is not always the case. The inclusion of off-shell contributions is essential to obtain an accurate Higgs signal normalization at the $1 \%$ precision level. For $g g \rightarrow H \rightarrow V V, V=W, Z, \mathcal{O}(5-10 \%)$ corrections occur due to an enhanced Higgs signal in the region $M_{V V}>2 M_{V}$, where also sizable Higgscontinuum interference occurs. We discuss how experimental selection cuts can be used to suppress this region in search channels where the Higgs mass cannot be reconstructed. We note that $H \rightarrow V V$ decay modes in non-gluon-fusion channels are similarly affected.
\end{abstract}

Keywords: Approximations; Higgs physics; hadron-hadron scattering.

PACS Nos.: 11.80.Fv, 14.80.Bn, 13.85.-t

email:n.kauer@rhul.ac.uk 


\section{Introduction}

The theoretical study of unstable particles has a long history. Initial work focused on an adequate field-theoretical definition of the mass and lifetime of an unstable particle [1-3]. At the time, a mass and width definition based on the position of the complex pole of the unstable particle propagator was also proposed [4]. A consistent quantum-fieldtheoretical formalism for the description of unstable particles with unitary, renormalizable and causal $S$-matrix was developed in Ref. [7]. Extending previous work to gauge theories, Refs. [8,9] proposed a perturbative expansion of the exact scattering amplitude about the complex pole of the unstable particle propagator and a subsequent consistent expansion in powers of the coupling constant. Thus obtaining an order by order gauge-invariant definition of the scattering amplitude in perturbation theory. The gauge-invariant [10] mass and width of the unstable particle in the corresponding pole scheme differ from the quantities in the commonly used on-shell scheme (see also Refs. [11,12]), which exhibit a gauge dependence in higher orders 1 The generalization of the complex pole approach to one-loop calculations with multiple, charged unstable particles has been discussed in Ref. [15].

In Refs. [16 20], the complex pole approach has been generalized using the effective field theory formalism. The effective theory is constructed by systematically expanding cross sections in powers of two parameters: the coupling constant $(\alpha)$ and a measure for the off-shellness of the unstable particle propagator $(\delta)$, which is of order $\Gamma / M$ in the region of validity, i.e. close to resonance.2 The effective field theory approach yields a gauge-invariant expansion that can in principle be truncated at arbitrarily high orders in $\alpha$ and $\delta$ and that permits an extension, for instance, to threshold regions.

An alternative approach that works more automatically and is expected to give precise numerical results in all kinematic situations, but is theoretically less rigorous, is the complex-mass scheme [23 25]. At tree level, it exploits the fact that the full amplitude (without self-energy resummation) is gauge independent and remains so if the mass of the unstable particle, $M$, is systematically replaced with $\sqrt{M^{2}-i M \Gamma}$, thus including the higher-order self-energy corrections that remove the real pole of the unstable particle propagator. Note that this procedure results, for instance, in a complex weak mixing angle: $\cos \theta_{W}=M_{W} / M_{Z}$. The introduced spurious terms are corrections of $\mathcal{O}(\Gamma / M)=\mathcal{O}(\alpha)$ in the resonant as well as nonresonant phase space regions. Beyond tree level, the complexmass scheme is implemented by splitting the real bare mass into a complex renormalized mass and a complex counterterm in the Lagrangian. The complete renormalization prescription is given in Ref. [24]. The complex-mass scheme has primarily been employed

\footnotetext{
${ }^{1}$ The gauge-independent definition of partial widths and branching ratios has been studied in Refs. [13, 14.

${ }^{2}$ In calculations, applying the method of regions [21,22] can be more convenient than the explicit procedure.
} 
in multi-leg calculations at tree and one-loop level 3 but can in principle be extended to higher orders. The theoretical weakness of the scheme is that the ad hoc introduction of complex masses violates unitarity. It is plausible that the unitarity-violating terms are of higher order, but a rigorous proof is still outstanding.

The outlined evolution of methods for perturbative calculations that involve unstable particles illustrates that despite the impressive progress no formalism has been developed yet that on the one hand has a rigorous field-theoretical foundation and on the other hand provides a practicable and efficient implementation which returns reliable results of the desired precision for all phenomenologically relevant observables. In this light, it is suggestive to revisit approximations where unstable particle states do not feature a continuous invariant mass spectrum, but are instead on-mass-shell.

The simplest such approximation is to treat unstable particles as stable external particles. The stable-particle approximation can be used to obtain results for inclusive observables with an expected uncertainty of $\mathcal{O}(\Gamma / M)$. But, it does not allow to calculate the differential cross section for processes that include the decay of the unstable particle. This is, however, essential for collider phenomenology, because typically only the decay products can be detected. Furthermore, as shown in Ref. [7], in a sound perturbative field theory unstable particles do not occur as external states 4

The zero-width approximation (ZWA) [26], a.k.a. narrow-width approximation, is a well-known on-shell approximation that is not affected by these shortcomings. It exploits the asymptotic equality of the squared modulus of the unstable (scalar) particle propagator with 4-momentum $q$ to $\pi /(M \Gamma) \delta\left(q^{2}-M^{2}\right)$ in the limit $\Gamma \rightarrow 0$. The Dirac delta function restricts the unstable particle to on-shell states without otherwise affecting the production and decay subprocesses. In general, an uncertainty of $\mathcal{O}(\Gamma / M)$ is expected for the ZWA. For many known and predicted unstable particles one finds that $\Gamma / M$ is of $\mathcal{O}(1 \%)$, which implies a ZWA error that is similar to other theoretical and experimental errors in collider experiments. The ZWA is therefore widely applicable. An exception are heavy Higgs bosons $\left(M_{H} \gtrsim 300 \mathrm{GeV}\right)$ : for a Standard Model $(\mathrm{SM})$ Higgs boson with mass of 300 $\mathrm{GeV}$, for instance, the expected ZWA uncertainty is of $\mathcal{O}(3 \%)$ and increases to $\mathcal{O}(20 \%)$ for $M_{H}=600 \mathrm{GeV}$. The ZWA is evidently not adequate for a heavy Higgs boson, which rather requires a detailed description of the lineshape (Higgs invariant mass distribution), which has been developed recently in Refs. [27, 28]. On the other hand, for the recently discovered [29, 30] Higgs boson with $M_{H} \approx 125 \mathrm{GeV}$, one has $\Gamma_{H} / M_{H}<10^{-4}$ (in the SM), which suggests an outstanding accuracy of the ZWA. However, as shown in Ref. [31] for inclusive cross sections and cross sections with experimental selection cuts, the ZWA is in general not adequate and the error estimate $\mathcal{O}\left(\Gamma_{H} / M_{H}\right)$ is not reliable for a light Higgs boson.

The review is organized as follows: In Sec. 2, a general discussion of the ZWA and

\footnotetext{
${ }^{3}$ Note that one-loop integrals with complex internal masses are required.

${ }^{4}$ The absence of asymptotic final states for unstable particles is plausible.
} 
its uncertainty is presented. Then, the ZWA inadequacy for a light Higgs boson signal is elucidated in Sec. 3, followed by a summary in Sec. 4.

\section{Zero-Width Approximation: Definition and Uncer- tainty}

Consider the squared modulus of the unstable scalar particle propagator with 4-momentum $q$, mass $M$ and width $\Gamma$,

$$
D\left(q^{2}, M, \Gamma\right)=\frac{1}{\left(q^{2}-M^{2}\right)^{2}+(M \Gamma)^{2}},
$$

where the fixed-width Breit-Wigner scheme has been used. We note that the field-theoretical definition of the on-shell mass $M$ and on-shell width $\Gamma$ of an unstable particle are not fully satisfactory and that the complex-pole scheme is theoretically better motivated (see Sec. 11). However, the propagator in the complex-pole scheme, $1 /\left(q^{2}-s_{\text {pole }}\right)$ with $s_{\text {pole }}=\mu^{2}-i \mu \gamma$ (see e.g. Ref. [27]), can be obtained to very high precision by substituting $M \rightarrow \mu$ and $\Gamma \rightarrow \gamma$ in Eq. (11) 5 The scheme choice does therefore not affect our discussion of the ZWA. The formal definition of the ZWA is based on the following expansion:

$$
D\left(q^{2}, M, \Gamma\right)=C \delta\left(q^{2}-M^{2}\right)+P V\left[\frac{1}{\left(q^{2}-M^{2}\right)^{2}}\right]+\sum_{n=0}^{N} c_{n}(\alpha) \delta_{n}\left(q^{2}-M^{2}\right),
$$

where $P V$ denotes the principal value (understood as a distribution), $\delta_{n}(x)$ is related to the $n$th derivative of the delta function by $\delta_{n}(x)=(-1)^{n} / n ! \delta^{(n)}(x)$, and the expansion is in terms of the coupling constant $\alpha$, to a given order $N$. The prefactor of the delta function is given by

$$
C=\frac{\pi}{M \Gamma}=\int_{-\infty}^{+\infty} d q^{2} D\left(q^{2}, M, \Gamma\right)
$$

In ZWA, only the first term of the expansion is taken into account. The ZWA hence factorizes the reaction into a production and a decay subprocess, joined by an on-shell unstable particle state.

The generalization of the ZWA to unstable particles with nonzero spin is straightforward, because the pole structure of the propagator is the same, and the expansion in Eq. (2) is similarly applicable. Note that in this form the ZWA preserves full spin and polarization correlations. One can take the factorization of production and decay subprocesses further and sum/average over the spin/polarization states of the intermediate on-shell state in order to fully decouple production and decay. As shown in Ref. [32], this procedure is

\footnotetext{
${ }^{5}$ The numerical difference between $D\left(q^{2}, \mu, \gamma\right)$ and the exact complex-pole scheme result is negligible in single precision.
} 
exact for total or sufficiently inclusive cross sections of arbitrary resonant processes with an on-shell intermediate state decaying via a cubic or quartic vertex. For less inclusive cross sections, the decorrelation approximation will cause an additional error. Note that branching ratios (BR) are defined and extracted from collider data via

$$
\mathrm{BR}_{d}=\frac{\Gamma_{d}}{\Gamma} \text { and } \sigma=\sigma_{p} \cdot \mathrm{BR}_{d},
$$

respectively, where $\Gamma_{d}(\Gamma)$ is the partial (total) decay width and $\sigma_{p}$ is the production cross section of the unstable particle in the stable-particle approximation. The right-hand side of Eq. (44) implies the application of the ZWA and decorrelation approximation. A process-independent branching ratio definition requires that production and decay are fully decoupled.

The uncertainty of the ZWA is typically of $\mathcal{O}(\Gamma / M)$. To be useful for high-energy scattering reactions, the ZWA uncertainty has to be comparable to other theoretical and experimental errors. Since for many known and predicted unstable particles $\Gamma / M$ is of $\mathcal{O}(1 \%)$, this is indeed the case. The ZWA is in particular widely used in the analysis of many-particle signatures due to Beyond-the-Standard-Model (BSM) physics 6 In this context, long decay chains frequently occur, and a repeated application of the ZWA suggests itself 7 Since application of the ZWA requires the corresponding intermediate unstable particle state and restricts it to the mass shell, its application implies that subresonant 8 and nonresonant amplitude contributions and off-shell effects are neglected 9 For sufficiently inclusive cross sections, these contributions are suppressed. The factorization into production and decay subprocesses is also convenient when higher-order corrections are included in calculations 10 In this case, in ZWA also non-factorizable corrections that connect production and decay subprocesses are neglected 11 Calculations for phenomenological studies are hence simplified in two ways: First, and most importantly, the number of contributing Feynman graphs is significantly reduced as well as the number of kinematic structures that need to be included when applying the multichannel Monte Carlo integration technique [38. Secondly, the dimensionality of the phase space integration is reduced. We note that state-of-the-art calculational techniques for amplitudes [39] and modern computer resources allow to include finite-width effects for processes of increasing complexity, in particular for SM processes and when BSM parameter space scans are not carried out.

An alternative interpretation of the ZWA is that the leading $q^{2}$-dependence of the differential cross section, i.e. the Breit-Wigner resonance, is integrated out. We illustrate this in Eqs. (5), (6) , and (7), where $s$ is the total 4-momentum squared, subscripts $p$ and

\footnotetext{
${ }^{6} \mathrm{~A}$ method to include finite-width effects in BSM event generators is described in Ref. 33.

${ }^{7}$ See Ref. 34 for an example.

${ }^{8}$ Subresonant Feynman graphs contain some, but not all unstable states that are to be treated in ZWA.

${ }^{9}$ Note that off-shell contributions are implicitly included. See Eqs. (33), (6) and (7).

${ }^{10}$ See Ref. 35 for an example.

${ }^{11}$ For sufficiently inclusive cross sections, the nonfactorizable corrections are of $\mathcal{O}(\alpha \Gamma / M)[36,37$.
} 
$d$ refer to production and decay factors, respectively, and the $M$ and $\Gamma$ dependence of $D\left(q^{2}, M, \Gamma\right)$ has been suppressed.

$$
\begin{aligned}
\sigma & =\frac{1}{2 s}\left[\int_{q_{\min }^{2}}^{q_{\max }^{2}} \frac{d q^{2}}{2 \pi}\left(\int d \phi_{p}\left|\mathcal{M}_{p}\left(q^{2}\right)\right|^{2} D\left(q^{2}\right) \int d \phi_{d}\left|\mathcal{M}_{d}\left(q^{2}\right)\right|^{2}\right)\right] \\
\sigma_{\mathrm{ZWA}} & =\frac{1}{2 s}\left(\int d \phi_{p}\left|\mathcal{M}_{p}\left(M^{2}\right)\right|^{2}\right)\left(\int_{-\infty}^{\infty} \frac{d q^{2}}{2 \pi} D\left(q^{2}\right)\right)\left(\int d \phi_{d}\left|\mathcal{M}_{d}\left(M^{2}\right)\right|^{2}\right) \\
\sigma_{\mathrm{ZWA}} & =\frac{1}{2 s}\left(\int d \phi_{p}\left|\mathcal{M}_{p}\left(M^{2}\right)\right|^{2}\right) \frac{1}{2 M \Gamma}\left(\int d \phi_{d}\left|\mathcal{M}_{d}\left(M^{2}\right)\right|^{2}\right)
\end{aligned}
$$

Evidently, even for a sufficiently small ratio $\Gamma / M$, the ZWA can be inadequate if a significant modification of the Breit-Wigner lineshape occurs. Such a modification can be induced by the $q^{2}$-dependence of production and decay factors or be due to interference with sub- or nonresonant amplitude contributions, i.e. corrections or backgrounds which are neglected in ZWA. Here, an important observation is that the Breit-Wigner distribution does not drop off nearly as fast as, for instance, a Gaussian. The relative contribution of the tail more than $n$ widths from the peak can be estimated as $1 /(n \pi)$, because [40]

$$
\int_{(M-n \Gamma)^{2}}^{(M+n \Gamma)^{2}} \frac{d q^{2}}{2 \pi} \frac{1}{\left(q^{2}-M^{2}\right)^{2}+(M \Gamma)^{2}} \approx \frac{1}{2 M \Gamma}\left(1-\frac{1}{n \pi}\right) .
$$

Note that the tail region with $\left|\left(q^{2}\right)^{1 / 2}-M\right|>5 \Gamma$ thus contributes more than $6 \%$ to the resonant cross section. Selection cuts or kinematical bounds (e.g. $m^{2}<q^{2}<s_{\max }$, where $m$ is the sum of the masses of the decay products, and $s_{\max }$ is the squared CMS collision energy) can introduce cutoffs, which are not taken into account if the ZWA is used. Eq. (8) can be used to estimate the associated error. Typically, kinematical bounds will be far from $M$ in terms of $\Gamma$, in which case the uncertainty is negligible.

Nevertheless, phenomenologically relevant cases where the ZWA error exceeds $\mathcal{O}(\Gamma / M)$ by one order of magnitude or more have been identified in the literature [31, 32, 41, 43]. A strongly enhanced uncertainty is possible if in ZWA kinematical threshold configurations occur. Consider, for example, a decay chain $A \rightarrow B,(C \rightarrow D, E)$. In this case, configurations where $\left|M_{C}-\left(M_{A}-M_{B}\right)\right|$ or $\left|M_{C}-\left(M_{D}+M_{E}\right)\right|$ is smaller than a few $\Gamma_{C}$ would be critical. Such configurations are phase space suppressed, and the decays will be rare. We note that almost degenerate particle masses are not unnatural in SM extensions, as evidenced by the SPS benchmark scenarios for SUSY searches [44. The occurring significant deviations between ZWA and off-shell results can be traced back to threshold factors $\beta\left(Q_{1}, Q_{2}\right)=\left(1-Q_{1}^{2} / Q_{2}^{2}\right)^{1 / 2}$, where $Q_{1,2}$ are (invariant) masses. The phase-space element contains such factors. For certain interaction types, e.g. scalar-fermion-antifermion, additional powers of $\beta$ are contributed by $|\mathcal{M}|^{2}$, which enlarges the phase space region where strongly enhanced deviations occur. The effect can be explained by a significant deformation of the Breit-Wigner lineshape (peak and tail) caused by powers of the $q^{2}$-dependent 


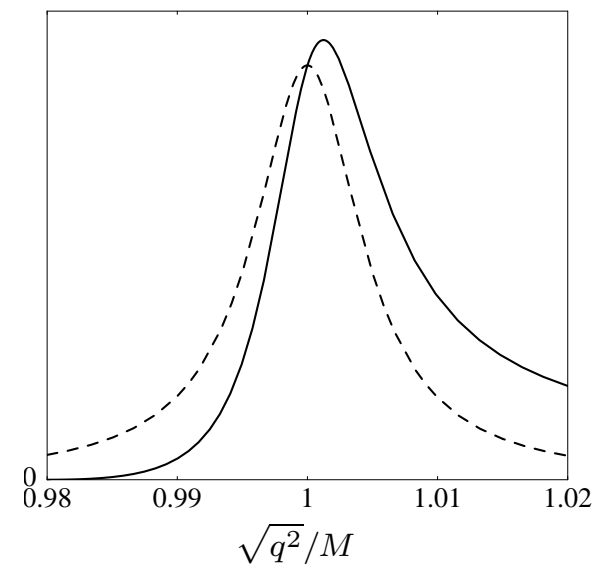

Figure 1: Breit-Wigner lineshape deformation caused by threshold factors when a decay daughter mass $m$ approaches the parent mass $M$. More specifically, the BreitWigner integrand $D\left(q^{2}, M, \Gamma\right)$ (dashed) and the integrand including threshold factors $D\left(q^{2}, M, \Gamma\right) f\left(q^{2}, m^{2}\right) / f\left(M^{2}, m^{2}\right)$ (solid) are shown in unspecified normalization for a decay via a scalar-fermion-antifermion interaction as functions of the invariant mass $\sqrt{q^{2}}$. $f(x, y)=(x-y)^{2} / x, \Gamma / M=1 \%$ and $m=M-2 \Gamma$. Further details can be found in Ref. [43].

threshold factors, as illustrated in Fig. 1. The $q^{2}$-dependence of the residual matrix element can cause strongly enhanced ZWA uncertainties even in the absence of degenerate mass configurations. An example is given in Sec. 3 ,

\section{Zero-Width Approximation Inadequacy for a Light Higgs Boson Signal}

A key objective of current particle physics research is the experimental confirmation of a theoretically consistent description of elementary particle masses. In the SM, this is achieved through the Higgs mechanism [45 49], which predicts the existence of one physical Higgs boson. A thorough examination of the recently discovered candidate SM Higgs boson [29, 30] with $M_{H} \approx 125 \mathrm{GeV}$ is in progress, and its properties are in agreement with theoretical expectations. No compelling deviations have been observed so far. In this situation, it is prudent to examine the accuracy of theoretical predictions for light Higgs production and decay that are used in experimental analyses. For light Higgs masses, the loop-induced gluon-fusion production $(g g \rightarrow H)$ dominates [50]. Next-to-leading order QCD corrections have been calculated in the heavy-top limit [51] and with finite $t$ and $b$ mass effects [52 54], and were found to be as large as 80-100\% at the Large Hadron Collider (LHC). This motivated the calculation of next-to-next-to-leading order QCD cor- 
rections [55 57] enhanced by soft-gluon resummation at next-to-next-to-leading logarithmic level [58,59] and beyond [60]. In addition to higher-order QCD corrections, electroweak corrections have been computed and found to be at the 1-5\% level [61 63]. Further references on all aspects of Higgs physics at the LHC can be found in Refs. [64, 65].

A comparison of the ZWA and finite-width Higgs propagator schemes in inclusive Higgs production and decay in gluon fusion was carried out in Refs. [66, 67] for Higgs masses between 120 and $800 \mathrm{GeV} 12$ Overall, good agreement with the expected uncertainty of $\mathcal{O}\left(\Gamma_{H} / M_{H}\right)$ was found. In particular, for light Higgs masses $\left(M_{H}<300 \mathrm{GeV}\right)$ a relatively small error of $\mathcal{O}(1 \%)$ was found [66, leading to the conclusion that the ZWA should be an adequate treatment for a light Higgs boson where the Higgs width is very small compared to its mass 67. Curiously, a closer inspection of the results for $M_{H}=120 \mathrm{GeV}$ reveal that the deviation between ZWA and fixed-width Breit-Wigner scheme $(0.5 \%)$ is two orders of magnitude larger than $\Gamma_{H} / M_{H}\left(4 \cdot 10^{-5}\right)$. Based on the discussion in Sec. 2, it is suggestive to interpret this as evidence for a significant deformation of the Breit-Wigner lineshape for a light Higgs boson. Such deformations were first predicted and thoroughly studied in Ref. [31. They can be traced back to the dependence of the Higgs decay amplitude $\mathcal{M}_{d}$ on the Higgs virtuality $q^{2}$ for different decay modes (cf. Eqs. (5) and (7)). One has, for instance 13

$$
\begin{aligned}
& \left|\mathcal{M}_{d}(H \rightarrow f \bar{f})\right|^{2} \sim M_{f}^{2} q^{2} \quad \text { for } \sqrt{q^{2}} \gtrsim 2 M_{f}, \\
& \left|\mathcal{M}_{d}(H \rightarrow V V)\right|^{2} \sim\left(q^{2}\right)^{2} \quad \text { for } \sqrt{q^{2}} \gtrsim 2 M_{V}
\end{aligned}
$$

for Higgs boson decays to fermions $f$ or weak bosons $V$. For the $H \rightarrow W W$ and $H \rightarrow Z Z$ decay modes of a light Higgs boson with resonance below the $V V$ threshold, a remarkable effect occurs above the $V V$ threshold (far away from the resonance peak): the leading $\left(q^{2}\right)^{-2}$ dependence of the off-shell squared Higgs propagator $|D|^{2}$ and the leading $\left(q^{2}\right)^{2}$ dependence of $\left|\mathcal{M}_{d}\right|^{2}$ largely compensate. The Higgs lineshape is therefore strongly enhanced for $\left(q^{2}\right)^{1 / 2}>2 M_{V}$ compared to the Breit-Wigner expectation, which is given by

$$
\left(\frac{d \sigma}{d M_{V V}}\right)_{\mathrm{ZWA}}=\sigma_{H, \mathrm{ZWA}} \frac{M_{H} \Gamma_{H}}{\pi} \frac{2 M_{V V}}{\left(M_{V V}^{2}-M_{H}^{2}\right)^{2}+\left(M_{H} \Gamma_{H}\right)^{2}} .
$$

The Breit-Wigner expectation $\left(H_{\mathrm{ZWA}}\right)$ and the enhanced off-shell distribution $\left(H_{\text {offshell }}\right)$ are illustrated in Figs. 2 and 3 using the $g g \rightarrow H \rightarrow W^{-} W^{+} \rightarrow \ell \bar{\nu}_{\ell} \bar{\ell} \nu_{\ell}$ and $g g \rightarrow H \rightarrow Z Z \rightarrow$ $\ell \bar{\ell} \nu_{\ell} \bar{\nu}_{\ell}$ processes, respectively. The differential cross section in the plateau-like finite-width tail is approximately two to three orders of magnitude smaller than in the resonance region. However, the plateau extends from the $V V$ threshold to beyond $600 \mathrm{GeV}$. The integrated

\footnotetext{
${ }^{12}$ We note that the pinch-technique approach yields a theoretically well-behaved Dyson-resummed Higgs boson propagator 68,69 , which does not suffer from unphysical absorptive effects, as seen in the comparison in Fig. 5 of Ref. [68.

${ }^{13}$ Here, $\gtrsim$ implies above, but not too far above threshold.
} 


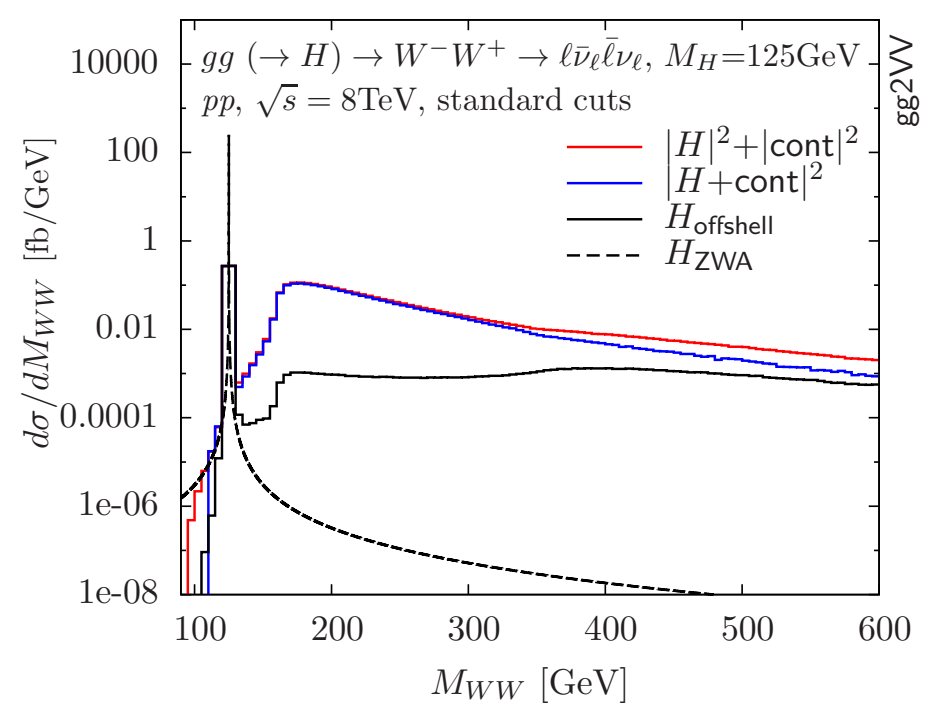

Figure 2: $M_{W W}$ distributions for $g g(\rightarrow H) \rightarrow W^{-} W^{+} \rightarrow \ell \bar{\nu}_{\ell} \bar{\ell} \nu_{\ell}$ in $p p$ collisions at $\sqrt{s}=8 \mathrm{TeV}$ for $M_{H}=125 \mathrm{GeV}$ and $\Gamma_{H}=0.004434 \mathrm{GeV}$ calculated at LO with gg2VV [70]. The ZWA distribution (black, dashed) as defined in Eq. (111) in the main text, the off-shell Higgs distribution (black, solid), the $d \sigma\left(\left|\mathcal{M}_{H}+\mathcal{M}_{\text {cont }}\right|^{2}\right) / d M_{W W}$ distribution (blue) and the $d \sigma\left(\left|\mathcal{M}_{H}\right|^{2}+\left|\mathcal{M}_{\text {cont }}\right|^{2}\right) / d M_{W W}$ distribution (red) are shown. Standard cuts are applied: $p_{T \ell}>20 \mathrm{GeV},\left|\eta_{\ell}\right|<2.5, p_{T}>30 \mathrm{GeV}, M_{\ell \ell}>12 \mathrm{GeV}$. Differential cross sections for a single lepton flavor combination are displayed. No flavor summation is carried out for charged leptons or neutrinos. Further details can be found in Ref. [31].

cross section in this region far from resonance thus contributes $\mathcal{O}(10 \%)$ to the total finitewidth cross section, more specifically, $16 \%$ and $37 \%$ in Figs. 2 and 3 , respectively. ZWA errors of $\mathcal{O}(10 \%)$ can therefore occur in $H \rightarrow V V$ decay modes, despite $\Gamma_{H} / M_{H}<10^{-4} 14$ We emphasize that $H \rightarrow V V$ modes in Higgs production channels other than gluon fusion also exhibit an enhanced off-shell tail, since the effect is caused by the decay amplitude.

Evidently, the ZWA caveat also applies to Monte Carlo generators that approximate off-shell effects with an ad hoc Breit-Wigner reweighting of the on-shell propagator (cf. Eq. (11)). Furthermore, the ZWA limitations are relevant for the extraction of Higgs couplings, which is initially being performed using the ZWA. The findings of Ref. 31 make clear that off-shell effects have to be included in future Higgs couplings analyses.

Above the $V V$ threshold, the $g g \rightarrow V V$ continuum background is large and sizable signal-background interference (see Fig. 4, left and right) occurs. Resonance-continuum interference in $g g(\rightarrow H) \rightarrow V V$ has been studied in Refs. [31,71,80] and for related pro-

\footnotetext{
${ }^{14}$ For $g g \rightarrow H \rightarrow \gamma \gamma$ the effect is drastically reduced and confined to the region $M_{\gamma \gamma}$ between $157 \mathrm{GeV}$ and $168 \mathrm{GeV}$, where the distribution is already five orders of magnitude smaller than in the resonance region.
} 


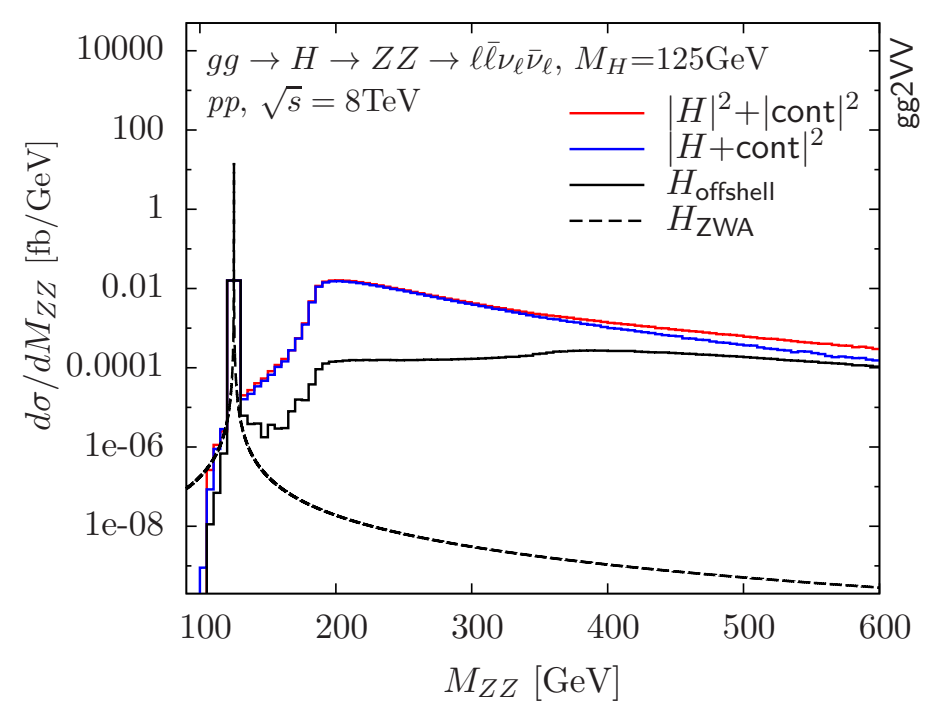

Figure 3: $M_{Z Z}$ distributions for $g g(\rightarrow H) \rightarrow Z Z \rightarrow \ell \bar{\ell} \nu_{\ell} \bar{\nu}_{\ell}$ for $M_{H}=125 \mathrm{GeV}$. Applied cuts: $p_{T \ell}>20 \mathrm{GeV},\left|\eta_{\ell}\right|<2.5,76 \mathrm{GeV}<M_{\ell \ell}<106 \mathrm{GeV}, \not p_{T}>10 \mathrm{GeV}$. Other details as in Fig. 2 ,

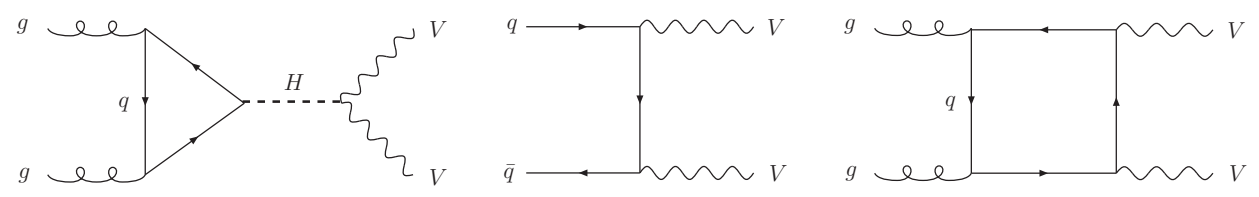

Figure 4: Representative Feynman graphs for the Higgs signal process (left) and the $q \bar{q}-$ (center) and $g g$-initiated (right) continuum background processes.

cesses in Refs. [81 87] 15 Due to the enhanced Higgs cross section above the $V V$ threshold, integrated cross sections can be affected by $\mathcal{O}(10 \%)$ signal-background interference effects, which are hence also displayed in Figs. 2 and 3.

In the vicinity of the Higgs resonance, finite-width and Higgs-continuum interference effects are negligible for $g g(\rightarrow H) \rightarrow V V$ if $M_{H} \ll 2 M_{V}$, as shown in Fig. 5 for $g g(\rightarrow$ $H) \rightarrow W^{-} W^{+} \rightarrow \ell \bar{\nu}_{\ell} \bar{\ell}_{\ell}$. For weak boson decays that permit the reconstruction of the Higgs invariant mass, the experimental procedure focuses on the Higgs resonance region and for $M_{H} \ll 2 M_{V}$ the enhanced off-shell region is thus typically excluded.

For $H \rightarrow V V$ channels that do not allow to reconstruct the Higgs invariant mass, the tail contribution can nevertheless be reduced significantly by means of optimized selection cuts. In Table 1, we demonstrate this for $g g(\rightarrow H) \rightarrow W^{-} W^{+} \rightarrow \ell \bar{\nu}_{\ell} \bar{\ell} \nu_{\ell}$. Here, the

\footnotetext{
${ }^{15}$ For studies of the $q \bar{q}$ and $g g$ continuum background (see Fig. 4 center and right), we refer the reader to Refs. [88, 95] and references therein.
} 


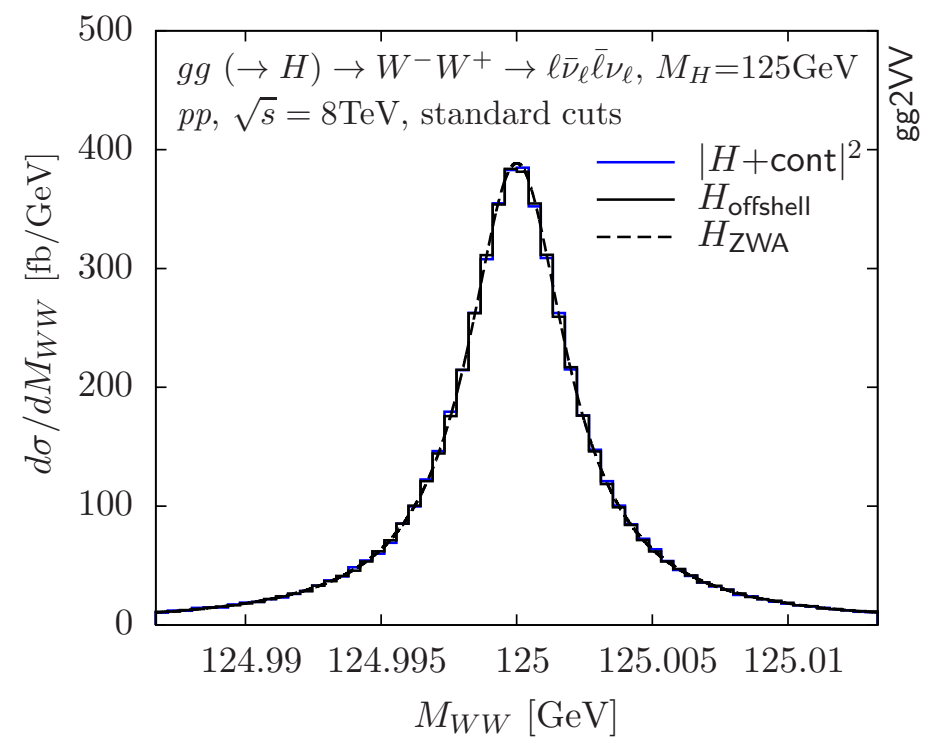

Figure 5: $M_{W W}$ distributions for $g g(\rightarrow H) \rightarrow W^{-} W^{+} \rightarrow \ell \bar{\nu}_{\ell} \bar{\ell}_{\ell}$ for $M_{H}=125 \mathrm{GeV}$. Off-shell and interference effects in the vicinity of the Higgs resonance are shown. Other details as in Fig. 2,

search selection has additional cuts, in particular an upper bound on the invariant mass of the observed dilepton system, which significantly reduce the contribution from the region with $M_{W W} \gg 2 M_{W}$. The result is a substantial mitigation of the off-shell (see Table 1) and interference effects (see Ref. [31]). As first noted in Ref. [76], a very effective means to suppress the tail contribution is provided by cuts on transverse mass observables [96], which are designed to have the physical mass of the decaying parent particle (the invariant mass in the off-shell case) as upper bound. For the process considered here, the best-performing transverse mass is defined by

$$
M_{T}=\sqrt{\left(M_{T, \ell \ell}+p_{T}\right)^{2}-\left(\mathbf{p}_{T, \ell \ell}+\boldsymbol{p}_{T}\right)^{2}} \text { with } M_{T, \ell \ell}=\sqrt{p_{T, \ell \ell}^{2}+M_{\ell \ell}^{2}} \text {. }
$$

$M_{T}$ distributions are shown in Fig. 6, which demonstrates that a $M_{T}<M_{H}$ cut strongly suppresses off-shell as well as interference effects. Table 1 shows that the application of this $M_{T}$ cut reduces the ZWA error to the sub-percent level. Note, however, that the cut on $M_{T}$ cannot completely eliminate the unwanted $M_{V V}>2 M_{V}$ contribution: $M_{T} \leq M_{V V}$ for all phase space configurations, and a small contamination remains. The efficiency of a transverse mass cut for $g g(\rightarrow H) \rightarrow Z Z \rightarrow \ell \bar{\ell} \nu_{\ell} \bar{\nu}_{\ell}$ is illustrated in Table 2. 
Table 1: Cross sections for $g g(\rightarrow H) \rightarrow W^{-} W^{+} \rightarrow \ell \bar{\nu}_{\ell} \bar{\ell} \nu_{\ell}$ and $M_{H}=125 \mathrm{GeV}$ with standard cuts, Higgs search cuts and additional cut on the transverse mass $M_{T}$ defined in Eq. (12) in the main text. Standard cuts: as in Fig. 2. Higgs search cuts: standard cuts and $M_{\ell \ell}<50 \mathrm{GeV}, \Delta \phi_{\ell \ell}<1.8$. The zero-width approximation (ZWA) and off-shell Higgs cross sections, the $g g$ continuum cross section and the sum of off-shell Higgs and continuum cross sections including interference are given. The accuracy of the ZWA and the impact of off-shell effects are assessed with $R=\sigma_{H, \mathrm{ZWA}} / \sigma_{H \text {,offshell. }}$ The integration error is given in brackets. Other details as in Fig. 2 .

\begin{tabular}{lccccc}
\hline \multicolumn{2}{c}{$g g(\rightarrow H) \rightarrow W^{-} W^{+} \rightarrow \ell \bar{\nu}_{\ell} \bar{\ell} \nu_{\ell}}$, & $\sigma[\mathrm{fb}]$, & $p p$, & $\sqrt{s}=8 \mathrm{TeV}$, & $M_{H}=125 \mathrm{GeV}$ \\
\hline selection cuts & $H_{\mathrm{ZWA}}$ & $H_{\text {offshell }}$ & cont & $\mid H_{\text {ofs }}+$ cont & 2 \\
\hline standard cuts & $2.707(3)$ & $3.225(3)$ & $10.493(5)$ & $12.241(8)$ & $0.839(2)$ \\
Higgs search cuts & $1.950(1)$ & $1.980(1)$ & $2.705(2)$ & $4.497(3)$ & $0.9850(7)$ \\
\hline $0.75 M_{H}<M_{T}<M_{H}$ & $1.7726(9)$ & $1.779(1)$ & $0.644(1)$ & $2.383(2)$ & $0.9966(8)$ \\
\hline
\end{tabular}

\section{Summary}

The evolution of methods for perturbative calculations that involve unstable particles has been reviewed. A general formalism that has a rigorous field-theoretical foundation and provides a practicable and efficient implementation which returns reliable results of the desired precision for all phenomenologically relevant observables is not yet known. This fact was used to motivate a review of on-mass-shell approximations. The zero-width approximation, a.k.a. narrow-width approximation, restricts the intermediate unstable particle state to the mass shell and, when combined with the decorrelation approximation, fully factorizes the production and decay subprocesses. Both approximations are implicitly ap-

Table 2: Cross sections for $g g(\rightarrow H) \rightarrow Z Z \rightarrow \ell \bar{\ell} \nu_{\ell} \bar{\nu}_{\ell}$ and $M_{H}=125 \mathrm{GeV}$ without and with transverse mass cut. Applied cuts: $p_{T \ell}>20 \mathrm{GeV},\left|\eta_{\ell}\right|<2.5,76 \mathrm{GeV}<M_{\ell \ell}<106$ $\mathrm{GeV}, \not p_{T}>10 \mathrm{GeV} . M_{T}$ is defined in Eq. (12) in the main text. Other details as in Table 1.

\begin{tabular}{lccccc}
\hline \multicolumn{2}{l}{$g(\rightarrow H) \rightarrow Z Z \rightarrow \ell \bar{\ell} \nu_{\ell} \bar{\nu}_{\ell}, \quad \sigma[\mathrm{fb}]}$, & $p p, \sqrt{s}=8 \mathrm{TeV}, \quad M_{H}=125 \mathrm{GeV}$ \\
\hline$M_{T}$ cut & $H_{\text {ZWA }}$ & $H_{\text {offshell }}$ & cont & $\mid H_{\text {ofs }}+$ cont $\left.\right|^{2}$ & $R$ \\
\hline none & $0.1593(2)$ & $0.2571(2)$ & $1.5631(7)$ & $1.6376(9)$ & $0.6196(7)$ \\
$M_{T}<M_{H}$ & $0.1593(2)$ & $0.1625(2)$ & $0.4197(5)$ & $0.5663(6)$ & $0.980(2)$ \\
\hline
\end{tabular}




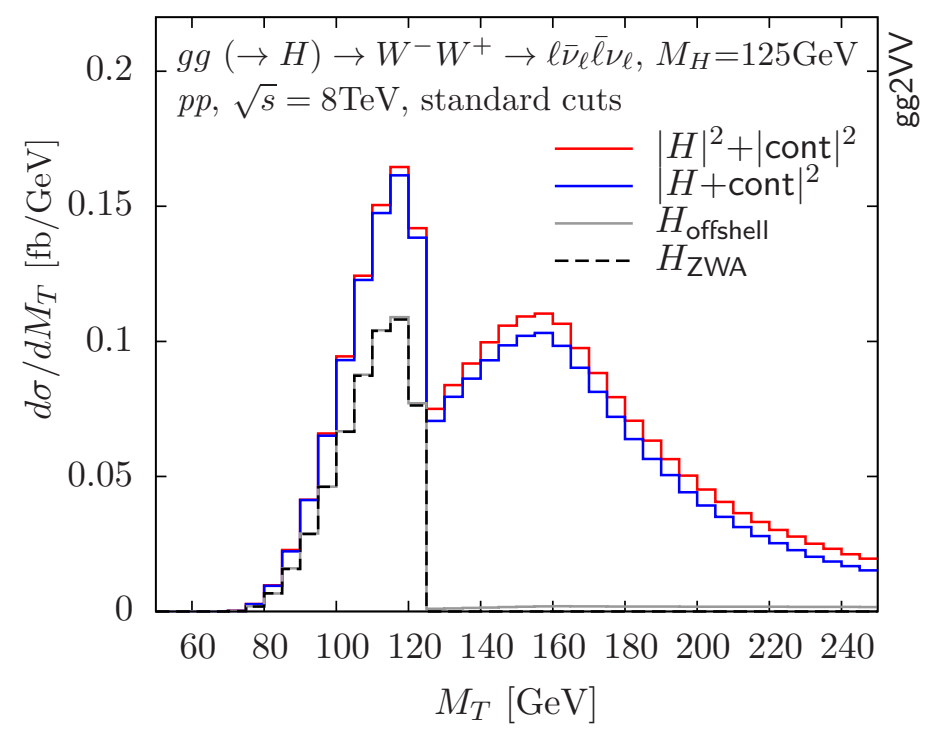

Figure 6: Transverse mass distributions for $g g(\rightarrow H) \rightarrow W^{-} W^{+} \rightarrow \ell \bar{\nu}_{\ell} \bar{\ell}_{\ell}$ and $M_{H}=125$ $\mathrm{GeV}$. Off-shell and interference effects in the region of the Higgs resonance and the $W$-pair threshold are shown. $M_{T}$ is defined in Eq. (12) in the main text. Other details as in Fig. 2.

plied when extracting branching ratios from collider data. The ZWA strongly reduces the complexity of calculations of cross sections for many-particle processes in Standard Model extensions and/or higher-order corrections. The uncertainty of the ZWA is typically of $\mathcal{O}(\Gamma / M)$, where $M(\Gamma)$ is the mass (width) of the unstable particle, but can be much larger in special cases where a significant modification of the Breit-Wigner lineshape occurs. Such a modification can be induced by the $q^{2}$-dependence of production and decay factors, where $q^{2}$ is the unstable particle virtuality, or be due to interference with sub- or nonresonant amplitude contributions, i.e. corrections or backgrounds which are neglected in ZWA. A strongly enhanced error is, for instance, possible if in ZWA kinematical threshold configurations occur.

A thorough examination of the recently discovered candidate SM Higgs boson is in progress, and its properties are in good agreement with theoretical expectations. It is thus prudent to examine the accuracy of theoretical predictions for light Higgs production and decay that are used in experimental analyses. For the SM Higgs boson with $M_{H} \approx$ $125 \mathrm{GeV}$, one has $\Gamma_{H} / M_{H}<10^{-4}$, which suggests an excellent accuracy of the ZWA. We have demonstrated that the ZWA is in general not adequate and the error estimate $\mathcal{O}\left(\Gamma_{H} / M_{H}\right)$ is not reliable for a light Higgs boson. The inclusion of off-shell contributions is essential to obtain an accurate Higgs signal normalization at the $1 \%$ precision level as well as correct kinematic distributions. ZWA deviations are particularly large for $g g \rightarrow H \rightarrow V V$ processes $(V=W, Z)$. To be more specific, without optimized selection cuts they are of 
$\mathcal{O}(5-10 \%)$. The ZWA caveat also applies to Monte Carlo generators that approximate offshell effects with an ad hoc Breit-Wigner reweighting of the on-shell propagator. Moreover, the ZWA limitations are relevant for the extraction of Higgs couplings, which is initially being performed using the ZWA. Our findings make clear that off-shell effects have to be included in future Higgs couplings analyses. The unexpected off-shell effect can be traced back to the dependence of the decay amplitude on the Higgs virtuality $q^{2}$. For $H \rightarrow V V$ decay modes one finds that the $\left(q^{2}\right)^{2}$ dependence of the squared modulus of the decay amplitude above the $V V$ threshold compensates the $q^{2}$-dependence of the Higgs propagator, which causes a strongly enhanced off-shell cross section up to invariant masses of about $600 \mathrm{GeV}$. We note that $H \rightarrow V V$ modes in Higgs production channels other than gluon fusion also exhibit an enhanced off-shell tail, since the effect is caused by the decay amplitude. The total $g g \rightarrow H \rightarrow V V$ cross section receives an $\mathcal{O}(10 \%)$ offshell correction and a similar correction due to signal-background interference. These effects are negligible in the vicinity of the Higgs resonance if $M_{H} \ll 2 M_{V}$. If the Higgs mass can be reconstructed exclusion of the problematic region above the $V V$ threshold is straightforward. For $H \rightarrow V V$ channels where this is not the case, the enhanced tail can be strongly suppressed by applying a $M_{T}<M_{H}$ cut on a suitable transverse mass observable $M_{T}$.

\section{Acknowledgments}

Important contributions of G. Passarino and financial support from HEFCE, STFC and the IPPP Durham are gratefully acknowledged.

\section{References}

[1] P. T. Matthews and A. Salam, Phys. Rev. 112, 283 (1958).

[2] P. T. Matthews and A. Salam, Phys. Rev. 115, 1079 (1959).

[3] R. Jacob and R. G. Sachs, Phys. Rev. 121, 350 (1961).

[4] R. E. Peierls, in The Proceedings of the 1954 Glasgow Conference on Nuclear and Meson Physics, eds. E. H. Bellamy and R. G. Moorhouse (Pergamon Press, 1955), p. 296.

[5] M. Lévy, Nuovo Cimento XIII, 115 (1959).

[6] R. J. Eden, P. V. Landshoff, D. I. Olive, and J. C. Polkinghorne, The Analytic S-Matrix (Cambridge University Press, 1966), p. 247.

[7] M. J. G. Veltman, Physica 29, 186 (1963). 
[8] R. G. Stuart, Phys. Lett. B 262, 113 (1991).

[9] A. Sirlin, Phys. Rev. Lett. 67, 2127 (1991).

[10] P. Gambino and P. A. Grassi, Phys. Rev. D 62, 076002 (2000) hep-ph/9907254.

[11] S. Willenbrock and G. Valencia, Phys. Lett. B 259, 373 (1991).

[12] B. A. Kniehl and A. Sirlin, Phys. Rev. Lett. 81, 1373 (1998) hep-ph/9805390.

[13] P. A. Grassi, B. A. Kniehl and A. Sirlin, Phys. Rev. Lett. 86, 389 (2001) hep-th/0005149.

[14] P. A. Grassi, B. A. Kniehl and A. Sirlin, Phys. Rev. D 65, 085001 (2002) hep-ph/0109228.

[15] A. Aeppli, G. J. van Oldenborgh and D. Wyler, Nucl. Phys. B 428, 126 (1994) hep-ph/9312212.

[16] M. Beneke, A. P. Chapovsky, A. Signer and G. Zanderighi, Phys. Rev. Lett. 93, 011602 (2004) hep-ph/0312331].

[17] M. Beneke, A. P. Chapovsky, A. Signer and G. Zanderighi, Nucl. Phys. B 686, 205 (2004) hep-ph/0401002.

[18] A. P. Chapovsky, V. A. Khoze, A. Signer and W. J. Stirling, Nucl. Phys. B 621, 257 (2002) hep-ph/0108190].

[19] P. Falgari, P. Mellor and A. Signer, Phys. Rev. D 82, 054028 (2010) arXiv:1007.0893 [hep-ph]].

[20] P. Falgari, A. S. Papanastasiou and A. Signer, arXiv:1303.5299 [hep-ph].

[21] M. Beneke and V. A. Smirnov, Nucl. Phys. B 522, 321 (1998) hep-ph/9711391.

[22] B. Jantzen, JHEP 1112, 076 (2011) [arXiv:1111.2589 [hep-ph]].

[23] A. Denner, S. Dittmaier, M. Roth and D. Wackeroth, Nucl. Phys. B 560, 33 (1999) hep-ph/9904472.

[24] A. Denner, S. Dittmaier, M. Roth and L. H. Wieders, Nucl. Phys. B 724, 247 (2005) [Erratum-ibid. B 854, 504 (2012)] hep-ph/0505042.

[25] A. Denner and S. Dittmaier, Nucl. Phys. Proc. Suppl. 160, 22 (2006) hep-ph/0605312].

[26] H. Pilkuhn, The Interactions of Hadrons (North-Holland, 1967). 
[27] S. Goria, G. Passarino and D. Rosco, Nucl. Phys. B 864, 530 (2012) arXiv:1112.5517 [hep-ph]].

[28] D. B. Franzosi, F. Maltoni and C. Zhang, arXiv:1211.4835 [hep-ph].

[29] G. Aad et al., Phys. Lett. B 716, 1 (2012) arXiv:1207.7214 [hep-ex]].

[30] S. Chatrchyan et al., Phys. Lett. B 716, 30 (2012) arXiv:1207.7235 [hep-ex]].

[31] N. Kauer and G. Passarino, JHEP 1208, 116 (2012) arXiv:1206.4803 [hep-ph]].

[32] C. F. Uhlemann and N. Kauer, Nucl. Phys. B 814, 195 (2009) arXiv:0807.4112 [hep$\mathrm{ph}]$.

[33] M. A. Gigg and P. Richardson, arXiv:0805.3037 [hep-ph].

[34] J. Reuter and D. Wiesler, Eur. Phys. J. C 73, 2355 (2013) arXiv:1212.5559 [hep-ph]].

[35] P. Falgari, C. Schwinn and C. Wever, JHEP 1301, 085 (2013) arXiv:1211.3408 [hep$\mathrm{ph}]$.

[36] K. Melnikov and O. I. Yakovlev, Phys. Lett. B 324, 217 (1994) hep-ph/9302311].

[37] V. S. Fadin, V. A. Khoze and A. D. Martin, Phys. Rev. D 49, 2247 (1994).

[38] F. A. Berends, R. Pittau and R. Kleiss, Nucl. Phys. B 424, 308 (1994) hep-ph/9404313.

[39] J. R. Andersen et al., arXiv:1003.1241 [hep-ph].

[40] N. Kauer and D. Zeppenfeld, Phys. Rev. D 65, 014021 (2002) hep-ph/0107181.

[41] D. Berdine, N. Kauer and D. Rainwater, Phys. Rev. Lett. 99, 111601 (2007) hep-ph/0703058.

[42] N. Kauer, Phys. Lett. B 649, 413 (2007) hep-ph/0703077].

[43] N. Kauer, JHEP 0804, 055 (2008) [arXiv:0708.1161 [hep-ph]].

[44] B. C. Allanach, M. Battaglia, G. A. Blair, M. S. Carena, A. De Roeck, A. Dedes, A. Djouadi, D. Gerdes et al., Eur. Phys. J. C 25, 113 (2002) hep-ph/0202233.

[45] P. W. Higgs, Phys. Lett. 12, 132 (1964).

[46] P. W. Higgs, Phys. Rev. Lett. 13, 508 (1964).

[47] P. W. Higgs, Phys. Rev. 145, 1156 (1966). 
[48] F. Englert and R. Brout, Phys. Rev. Lett. 13, 321 (1964).

[49] G. S. Guralnik, C. R. Hagen and T. W. B. Kibble, Phys. Rev. Lett. 13, 585 (1964).

[50] H. M. Georgi, S. L. Glashow, M. E. Machacek and D. V. Nanopoulos, Phys. Rev. Lett. 40, 692 (1978).

[51] S. Dawson, Nucl. Phys. B 359, 283 (1991).

[52] A. Djouadi, M. Spira and P. M. Zerwas, Phys. Lett. B 264, 440 (1991).

[53] D. Graudenz, M. Spira and P. M. Zerwas, Phys. Rev. Lett. 70, 1372 (1993).

[54] M. Spira, A. Djouadi, D. Graudenz and P. M. Zerwas, Nucl. Phys. B 453, 17 (1995) hep-ph/9504378.

[55] R. V. Harlander and W. B. Kilgore, Phys. Rev. Lett. 88, 201801 (2002) hep-ph/0201206.

[56] C. Anastasiou and K. Melnikov, Nucl. Phys. B 646, $220 \quad(2002)$ arXiv:hep-ph/0207004.

[57] V. Ravindran, J. Smith and W. L. van Neerven, Nucl. Phys. B 665, 325 (2003) arXiv:hep-ph/0302135.

[58] S. Catani, D. de Florian, M. Grazzini and P. Nason, JHEP 0307, 028 (2003) hep-ph/0306211.

[59] D. de Florian, G. Ferrera, M. Grazzini and D. Tommasini, JHEP 1111, 064 (2011) arXiv:1109.2109 [hep-ph]].

[60] S. Moch and A. Vogt, Phys. Lett. B 631, 48 (2005) hep-ph/0508265.

[61] A. Djouadi and P. Gambino, Phys. Rev. Lett. 73, 2528 (1994) hep-ph/9406432.

[62] S. Actis, G. Passarino, C. Sturm and S. Uccirati, Phys. Lett. B 670, 12 (2008) arXiv:0809.1301 [hep-ph]].

[63] C. Anastasiou, R. Boughezal and F. Petriello, JHEP 0904, 003 (2009) arXiv:0811.3458 [hep-ph]].

[64] S. Dittmaier et al., arXiv:1101.0593 [hep-ph].

[65] S. Dittmaier et al., arXiv:1201.3084 [hep-ph].

[66] C. Anastasiou, S. Buehler, F. Herzog and A. Lazopoulos, JHEP 1112, 058 (2011) arXiv:1107.0683 [hep-ph]]. 
[67] C. Anastasiou, S. Buehler, F. Herzog and A. Lazopoulos, JHEP 1204, 004 (2012) arXiv:1202.3638 [hep-ph]].

[68] J. Papavassiliou and A. Pilaftsis, Phys. Rev. D 58, 053002 (1998) hep-ph/9710426.

[69] J. Papavassiliou and A. Pilaftsis, Phys. Rev. Lett. 80, 2785 (1998) hep-ph/9710380.

[70] http://gg2VV.hepforge.org/

[71] E. W. N. Glover and J. J. van der Bij, Phys. Lett. B 219, 488 (1989).

[72] E. W. N. Glover and J. J. van der Bij, Nucl. Phys. B 321, 561 (1989).

[73] M. H. Seymour, Phys. Lett. B 354, 409 (1995) hep-ph/9505211].

[74] T. Binoth, M. Ciccolini, N. Kauer and M. Kramer, JHEP 0612, 046 (2006) hep-ph/0611170].

[75] E. Accomando, Phys. Lett. B 661, 129 (2008) [arXiv:0709.1364 [hep-ph]].

[76] J. M. Campbell, R. K. Ellis and C. Williams, JHEP 1110, 005 (2011) arXiv:1107.5569 [hep-ph]].

[77] N. Kauer, arXiv:1201.1667 [hep-ph].

[78] G. Passarino, JHEP 1208, 146 (2012) [arXiv:1206.3824 [hep-ph]].

[79] F. Campanario, Q. Li, M. Rauch and M. Spira, arXiv:1211.5429 [hep-ph].

[80] M. Bonvini, F. Caola, S. Forte, K. Melnikov and G. Ridolfi, arXiv:1304.3053 [hep-ph].

[81] L. J. Dixon and M. S. Siu, Phys. Rev. Lett. 90, 252001 (2003) hep-ph/0302233].

[82] L. J. Dixon and Y. Sofianatos, Phys. Rev. D 79, 033002 (2009) arXiv:0812.3712 [hep-ph]].

[83] E. Accomando, D. Becciolini, S. De Curtis, D. Dominici, L. Fedeli and C. ShepherdThemistocleous, Phys. Rev. D 85, 115017 (2012) [arXiv:1110.0713 [hep-ph]].

[84] S. P. Martin, Phys. Rev. D 86, 073016 (2012) arXiv:1208.1533 [hep-ph]].

[85] D. de Florian, N. Fidanza, R. J. Hernandez-Pinto, J. Mazzitelli, Y. R. Habarnau and G. F. R. Sborlini, arXiv:1303.1397 [hep-ph].

[86] S. P. Martin, arXiv:1303.3342 [hep-ph].

[87] E. Accomando, D. Becciolini, A. Belyaev, S. Moretti and C. Shepherd-Themistocleous, arXiv:1304.6700 [hep-ph]. 
[88] J. M. Campbell and R. K. Ellis, Phys. Rev. D 60, 113006 (1999) arXiv:hep-ph/9905386.

[89] T. Binoth, M. Ciccolini, N. Kauer and M. Kramer, JHEP 0503, 065 (2005) hep-ph/0503094.

[90] T. Binoth, N. Kauer and P. Mertsch, arXiv:0807.0024 [hep-ph].

[91] J. M. Campbell, R. K. Ellis and C. Williams, JHEP 1107, 018 (2011) arXiv:1105.0020 [hep-ph]].

[92] T. Melia, P. Nason, R. Rontsch and G. Zanderighi, JHEP 1111, 078 (2011) arXiv:1107.5051 [hep-ph]].

[93] R. Frederix, S. Frixione, V. Hirschi, F. Maltoni, R. Pittau and P. Torrielli, JHEP 1202, 099 (2012) [arXiv:1110.4738 [hep-ph]].

[94] T. Melia, K. Melnikov, R. Rontsch, M. Schulze and G. Zanderighi, JHEP 1208, 115 (2012) [arXiv:1205.6987 [hep-ph]].

[95] P. Agrawal and A. Shivaji, Phys. Rev. D 86, 073013 (2012) [arXiv:1207.2927[hep-ph]].

[96] A. J. Barr, B. Gripaios and C. G. Lester, JHEP 0907, 072 (2009) arXiv:0902.4864 [hep-ph]]. 